\title{
ON A CERTAIN FUNCTIONAL EQUATION IN THE ALGEBRA OF POLYNOMIALS WITH COMPLEX COEFFICIENTS
}

\author{
E. MUHAMADIEV
}

Received 26 June 2005; Accepted 1 July 2005

Many analytical problems can be reduced to determining the number of roots of a polynomial in a given disc. In turn, the latter problem admits further reduction to the generalized Rauss-Hurwitz problem of determining the number of roots of a polynomial in a semiplane. However, this procedure requires complicated coefficient transformations. In the present paper we suggest a direct method to evaluate the number of roots of a polynomial with complex coefficients in a disc, based on studying a certain equation in the algebra of polynomials. An application for computing the rotation of plane polynomial vector fields is also given.

Copyright (c) 2006 E. Muhamadiev. This is an open access article distributed under the Creative Commons Attribution License, which permits unrestricted use, distribution, and reproduction in any medium, provided the original work is properly cited.

\section{Functional equations: basic properties of solutions}

Let

$$
\begin{array}{cl}
f(z)=a_{0}+a_{1} z+\cdots+a_{n} z^{n}, & a_{n} \neq 0, \\
F(z)=b_{0}+b_{1} z+\cdots+b_{n} z^{n}+b_{n+1} z^{n+1}, & b_{0} \neq 0, b_{n+1} \neq 0,
\end{array}
$$

be polynomials with complex coefficients $a_{0}, a_{1}, \ldots, a_{n}$ and $b_{0}, b_{1}, \ldots, b_{n+1}$ of degree $n$ and $n+1$, respectively. Assume the polynomials $f$ and $F$ to satisfy the functional equation

$$
(a+b z) f(z)+(c+d z) f^{*}(z)=F(z)
$$

where $a, b, c, d$ are certain complex numbers and the polynomial $f^{*}$ is defined by

$$
f^{*}(z)=\bar{a}_{0} z^{n}+\bar{a}_{1} z^{n-1}+\cdots+\bar{a}_{n}=z^{n} \overline{f\left(\frac{1}{\bar{z}}\right)} .
$$


2 On a certain functional equation in the algebra of polynomials

Consider along with (1.3) the following functional equation:

$$
g(z) \cdot f(z)=(\bar{a} z+\bar{b}) F(z)-(c+d z) F^{*}(z),
$$

where

$$
\begin{gathered}
g(z)=\alpha z^{2}+\beta z+\bar{\alpha}, \quad \alpha=\bar{a} b-\bar{c} d, \beta=|a|^{2}+|b|^{2}-|c|^{2}-|d|^{2}, \\
F^{*}(z)=\bar{b}_{0} z^{n+1}+\bar{b}_{1} z^{n}+\cdots+\bar{b}_{n+1} .
\end{gathered}
$$

Lemma 1.1. If the polynomials $f$ and $F$ satisfy the functional equation (1.3), then they also satisfy the functional equation (1.5).

Conversely, if at least one of the numbers $\alpha, \beta$ is different from zero and $f, F$ satisfy (1.5), then they satisfy (1.3) as well.

Proof. Let polynomials (1.1) and (1.2) satisfy (1.3). By the definition of $f^{*}$ and $F^{*}$, one has

$$
(\bar{a} z+\bar{b}) f^{*}(z)+(\bar{c} z+\bar{d}) f(z)=F^{*}(z) .
$$

Multiplying (1.3) (resp., (1.8)) by $(\bar{a} z+\bar{b})$ (resp., by $(c+d z))$ and taking the difference of the obtained expressions, one obtains (1.5), where the coefficients $\alpha$ and $\beta$ are defined by (1.6). Thus the first implication is established.

Conversely, assume that $f$ and $F$ satisfy (1.5), and at least one of $\alpha, \beta$ is different from zero. Since the coefficient $\beta$ of $g$ is real (cf. [4]), one has

$$
z^{2} \overline{g\left(\frac{1}{\bar{z}}\right)}=g(z)
$$

Therefore, it follows from (1.5) that

$$
g(z) f^{*}(z)=(a+b z) F^{*}(z)-(\bar{c} z+\bar{d}) F(z) .
$$

Multiplying (1.5) (resp., (1.10)) by $(a+b z)$ (resp., by $(c+d z))$ and summing up the obtained expressions, one arrives at the following equality:

$$
g(z)\left[(a+b z) f(z)+(c+d z) f^{*}(z)\right]=g(z) F(z) .
$$

Since $g(z) \not \equiv 0$ and the algebra of polynomials does not contain zero divisors, it follows that $f$ and $F$ satisfy (1.3).

The lemma is completely proved.

Assume that the polynomials $f$ and $F$ satisfy (1.3). It follows from (1.1)-(1.3) that (1.3) is equivalent to the following system:

$$
a a_{k}+c \bar{a}_{n-k}+b a_{k-1}+d \bar{a}_{n-k+1}=b_{k}, \quad k=0,1, \ldots, n+1,
$$

where we put $a_{k}=0$ for $k<0$ and $k>n$. 
Similarly, (1.5) is equivalent to the following system:

$$
\bar{\alpha} a_{k}+\beta a_{k-1}+\alpha a_{k-2}=\bar{a} b_{k-1}-d \bar{b}_{n-k+2}+\bar{b} b_{k}-c \bar{b}_{n-k+1}, \quad k=0,1, \ldots, n+2,
$$

where $a_{k}=b_{k}=0$ for $k<0$ and $a_{k}=b_{k+1}=0$ for $k>n$.

Thus, under the assumption that $a, b, c$, and $d$ satisfy the condition $|\alpha|+|\beta|>0$, system (1.3) is equivalent to (1.12) as well as to (1.13).

Below we will list some properties of solutions to (1.3).

(1) (a) The coefficients $a, b, c, d$ along with the polynomial $f$ determine the polynomial $F$ uniquely.

(b) If the polynomials $f$ and $F$ are defined by (1.1) and (1.2) and satisfy (1.3), then the coefficients $a, b, c, d$ satisfy the conditions

$$
|a|+|c|>0, \quad|b|+|d|>0
$$

(c) If a collection $(a, b, c, d, f, F)$ of numbers $a, b, c, d$, and polynomials $f, F$ satisfy (1.3), then so is the collection $(\lambda a, \lambda b, \bar{\lambda} c, \bar{\lambda} d, f / \lambda, F)$ for any complex number $\lambda \neq 0$.

(2) Given a polynomial $F$ and numbers $a, b, c$, and $d$, satisfying $|\alpha|+|\beta|>0$, there exists a unique $f$ satisfying (1.3). Indeed, if $\alpha \neq 0$, then the first $n+1$ equations of system (1.13) completely determine the coefficients $a_{0}, a_{1}, \ldots, a_{n}$ of the polynomial $f$. If, however, $\alpha=0$ and $\beta \neq 0$, then all the coefficients $a_{0}, a_{1}, \ldots, a_{n}$ of the polynomial $f$ are completely determined by $n+1$ equations of system (1.13) starting with the second one.

(3) It follows from (1.5) that the roots of $g(z)$ turn out to be the roots of $G(z)=$ $(\bar{a} z+\bar{b}) F(z)-(c+d z) F^{*}(z)$. Also, if $\alpha \neq 0$ and $\beta^{2} \neq 4|\alpha|^{2}$, then $z_{0}, z_{1}=1 / \bar{z}_{0}$, where

$$
z_{0}=\frac{-\beta+\sqrt{\beta^{2}-4|\alpha|^{2}}}{2 \alpha},
$$

are the roots of $g(z)$. Therefore, $F$ and the coefficients $a, b, c, d$ are connected by the following relations:

$$
\left(\bar{a} z_{j}+\bar{b}\right) F\left(z_{j}\right)-\left(c+d z_{j}\right) F^{*}\left(z_{j}\right)=0, \quad j=0,1 .
$$

If $\alpha \neq 0$ and $\beta^{2}=4|\alpha|^{2}$, then $z_{1}=z_{0}$ is a multiple root of $g(z)$, and, therefore,

$$
\begin{gathered}
\left(\bar{a} z_{0}+\bar{b}\right) F\left(z_{0}\right)-\left(c+d z_{0}\right) F^{*}\left(z_{0}\right)=0, \\
\left(\bar{a} z_{0}+\bar{b}\right) F^{\prime}\left(z_{0}\right)+\bar{a} F\left(z_{0}\right)-\left(c+d z_{0}\right) F^{*}\left(z_{0}\right)-d F^{*}\left(z_{0}\right)=0 .
\end{gathered}
$$

Finally, if $\alpha=0$ and $\beta \neq 0$, then the linear function $g(z)=\beta z$ has the only root $z_{0}=0$. Hence it follows from (1.5) (see also (1.10)) that

$$
\begin{aligned}
& \bar{b} F(0)-c F^{*}(0)=0, \\
& \bar{d} F(0)-a F^{*}(0)=0 .
\end{aligned}
$$


4 On a certain functional equation in the algebra of polynomials

\section{Functional equations: solubility conditions}

Given the polynomial (1.2), consider the solubility problem for the functional equation (1.3) with respect to unknown coefficients $a, b, c, d$ and a polynomial $f$. To treat the above problem, we will use the necessary conditions for the solubility of (1.3) given by (3.1)-(3.8) (depending on whether the root $z_{0}$ of $g(z)$ satisfies $0<\left|z_{0}\right|<1,\left|z_{0}\right|=1$, or $\left.z_{0}=0\right)$.

Assume $z_{0}$ and $z_{1}$ to be given and consider system (1.16) with respect to unknown $a$, $b, c, d$. We will try to find a solution to (1.16) in such a way that (1.3) will have a solution with respect to $f$. Also, given $z_{0}$, we will follow the same way regarding system (1.17).

To describe the solubility conditions for the functional equation (1.3), it is convenient to introduce the notion of a regular point.

A point $z$ is called regular with respect to the polynomial $F$ if the following conditions are satisfied:

$$
\begin{gathered}
F(z) \cdot F^{*}(z) \neq 0, \quad|F(z)| \neq\left|F^{*}(z)\right|, \quad \text { for }|z| \neq 1, \\
(n+1)|F(z)|^{2} \neq 2 \mathfrak{R}\left[\overline{F(z)} F^{\prime}(z) z\right], \quad \text { for }|z|=1 .
\end{gathered}
$$

Observe that the notion of a regular point is introduced with respect to the unit circle. It follows immediately from the definition of a regular point that if $z_{0}$ is regular, then so is $z_{1}=1 / \bar{z}_{0}$ and vice versa.

According to the definition of polynomial $F^{*}$, the rational function

$$
A(z)=\frac{F^{*}(z)}{F(z)}
$$

satisfies the identity

$$
\overline{A(z)} \cdot A\left(\frac{1}{\bar{z}}\right) \equiv 1
$$

In addition, $|A(z)| \neq 1$ for all regular points $z,|z| \neq 1$.

Assume that $z_{0}$ is a regular point of $F, A_{0}=A\left(z_{0}\right), A_{1}=1 / \bar{A}_{0}$, and $\sigma_{0}, \sigma_{1}$ are arbitrary complex numbers. Consider the linear system

$$
\begin{array}{cl}
\bar{a} z_{0}+\bar{b}=\sigma_{0} A_{0}, & \bar{a}+\bar{b} \overline{z_{0}}=\sigma_{1} A_{1}, \\
c+d z_{0}=\sigma_{0}, & c \bar{z}_{0}+d=\sigma_{1},
\end{array}
$$

with unknown $a, b, c$, and $d$. It should be pointed out that any solution to system (2.4) is also a solution to (1.16) for $z_{0} \neq 0$, as well as a solution to (1.18) for $z_{0}=0$.

For $\left|z_{0}\right|<1$ system, (2.4) has the unique solution

$$
\begin{array}{cc}
\bar{a}\left(1-\left|z_{0}\right|^{2}\right)=\sigma_{1} A_{1}-\bar{z}_{0} \sigma_{0} A_{0}, & \bar{b}\left(1-\left|z_{0}\right|^{2}\right)=\sigma_{0} A_{0}-\bar{z}_{0} \sigma_{1} A_{1}, \\
c\left(1-\left|z_{0}\right|^{2}\right)=\sigma_{0}-z_{0} \sigma_{1}, \quad d\left(1-\left|z_{0}\right|^{2}\right)=\sigma_{1}-\bar{z}_{0} \sigma_{0} .
\end{array}
$$


With the above $a, b, c$, and $d$ on hands, the coefficients $\alpha, \beta$ from formula (1.6) satisfy the equalities

$$
\begin{aligned}
& \alpha\left(1-\left|z_{0}\right|^{2}\right)=\bar{z}_{0}\left(\left|A_{1}\right|^{2}-1\right)\left(\left|\sigma_{0} A_{0}\right|^{2}-\left|\sigma_{1}\right|^{2}\right), \\
& \beta\left(1-\left|z_{0}\right|^{2}\right)=-\left(\left|A_{1}\right|^{2}-1\right)\left(\left|\sigma_{0} A_{0}\right|^{2}-\left|\sigma_{1}\right|^{2}\right) .
\end{aligned}
$$

Theorem 2.1. Let $z_{0}$ with $\left|z_{0}\right|<1$ be a regular point of the polynomial $F$, and assume the numbers $\sigma_{0}$ and $\sigma_{1}$ to satisfy the condition

$$
\left|\sigma_{0} A_{0}\right| \neq\left|\sigma_{1}\right|
$$

Let, further, $a, b, c$, and $d$ be defined by (2.5). Then the functional equation (1.3) admits a solution $f(z)$.

Proof. To begin with, consider the case $z_{0} \neq 0$. It follows from (2.8) and (2.6) that $\alpha \neq 0$. Formulae (2.4) and (2.3) provide that $z_{0}$ and $z_{1}=1 / \bar{z}_{0}$ are the roots of the polynomial $g(z)(1.6)$ :

$$
\begin{gathered}
g\left(z_{0}\right)=\left(a+b z_{0}\right)\left(\bar{a} z_{0}+\bar{b}\right)-\left(c+d z_{0}\right)\left(\bar{c} z_{0}+\bar{d}\right)=\bar{\sigma}_{1} \bar{A}_{1} \sigma_{0} A_{0}-\sigma_{0} \bar{\sigma}_{1}=\bar{\sigma}_{1} \sigma_{0}-\sigma_{0} \bar{\sigma}_{1}=0, \\
g\left(z_{1}\right)=z_{1}^{2} \overline{g\left(z_{0}\right)}=0 .
\end{gathered}
$$

On the other hand, the numbers $z_{0}$ and $z_{1}$ are the roots of the polynomial

$$
G(z)=(\bar{a} z+\bar{b}) F(z)-(c+d z) F^{*}(z) .
$$

Therefore, by the Bezout theorem, the rational function

$$
f(z)=\frac{G(z)}{g(z)}
$$

is, in fact, a polynomial satisfying the functional equation (1.5). Now the statement of the theorem in the considered case follows from Lemma 1.1.

Assume now $z_{0}=0$. The condition (2.8) and equalities (2.6), (2.7) yield $\alpha=0$ and $\beta \neq$ 0 , that is, $g(z)=\beta z$ is a linear function. In addition, $z=0$ is a root of the polynomial $G(z)$. Therefore, $f(z)=G(z) / g(z)$ is a polynomial satisfying (1.5), and again the statement of the theorem in the considered case follows from Lemma 1.1.

Theorem 2.1 is completely proved.

Assume now that a regular point $z_{0}$ of the polynomial $F$ belongs to the unit circle $|z|=1$. Given numbers $c$ and $d$, consider system (1.18) with unknown $a, b$. By solving system (1.18) one obtains

$$
\bar{a} F^{2}=\Delta \cdot c+\left(F F^{*}+z \Delta\right) d, \quad \bar{b} F^{2}=\left(F F^{*}-z \Delta\right) c-z^{2} \Delta d,
$$


6 On a certain functional equation in the algebra of polynomials

where

$$
\begin{gathered}
\Delta=z^{n} \Delta_{0}, \quad \Delta_{0}=(n+1)|F|^{2}-2 \mathfrak{R}\left[\bar{F} F^{\prime} z\right], \\
F=F(z), \quad F^{\prime}=F^{\prime}(z), \quad F^{*}=F^{*}(z), \quad z=z_{0} .
\end{gathered}
$$

Take the coefficients $a, b, c$, and $d$ satisfying (2.12) and define the polynomial $g(z)$ by means of formula (1.6). Formula (2.12) provides the following relations for $\alpha$ and $\beta$ :

$$
\begin{aligned}
& z \alpha|F|^{4}=\Delta_{0}\left[|F|^{2}\left(|c|^{2}-|d|^{2}\right)-\Delta_{0}|c+z d|^{2}\right], \\
& \beta|F|^{4}=2 \Delta_{0}\left[\Delta_{0}|c+z d|^{2}-|F|^{2}\left(|c|^{2}-|d|^{2}\right)\right] .
\end{aligned}
$$

Theorem 2.2. Let $z_{0},\left|z_{0}\right|=1$ be a regular point of the polynomial $F$ and let the numbers $c$, $d$ satisfy the condition:

$$
\Delta_{0}\left|c+z_{0} d\right|^{2} \neq\left|F\left(z_{0}\right)\right|^{2}\left(|c|^{2}-|d|^{2}\right)
$$

If the coefficients $a, b, c$, and $d$ satisfy relation (2.12), then the functional equation (1.3) has the unique solution $(a, b, c, d, f)$.

Proof. It follows from (1.6) and (2.15)-(3.1) that $\beta=-2 \alpha z_{0} \neq 0$. Since the coefficient $\beta$ is real and $z_{0} \bar{z}_{0}=1$, one obtains the equality $\bar{\alpha}=\alpha z_{0}^{2}$, that is, $g(z) \equiv \alpha\left(z-z_{0}\right)^{2}$. At the same time, $z_{0}$ is a multiple root to the polynomial $G(z)=(\bar{a} z+\bar{b}) F(z)-(c+d z) F^{*}(z)$. Therefore, the rational function $f(z)=G(z) / g(z)$ is, in fact, a polynomial satisfying the functional equation (1.5). To complete the proof of Theorem 2.1 it remains to apply Lemma 1.1. The theorem follows.

Combining Theorems 2.1 and 2.2 with property (2) of solutions to (1.3) one can effectively compute the numbers $a, b, c, d$ and the coefficients $a_{0}, a_{1}, \ldots, a_{n}$ of the polynomial $f$. Indeed, assume, for instance, that $z=0$ is a regular point of the polynomial $F$. Then the regularity condition for the point $z=0$ along with condition (2.8) take the form

$$
b_{0} \cdot b_{n+1} \neq 0, \quad\left|b_{0}\right| \neq\left|b_{n+1}\right|, \quad\left|c b_{n+1}\right| \neq\left|d b_{0}\right|
$$

also, the equalities (2.6) and (2.7) take the form

$$
\bar{a} b_{n+1}=d \bar{b}_{0}, \quad \bar{b} b_{0}=c \bar{b}_{n+1} .
$$

In this case the leading coefficient $\alpha$ of the polynomial $g(z)$, that is determined by $a$, $b, c, d$, is equal to zero. Hence, in order to determine unknown coefficients $a_{0}, a_{1}, \ldots, a_{n}$ from system (1.13), one has

$$
\begin{gathered}
0=\bar{b} b_{0}-c \bar{b}_{n+1}, \\
\beta a_{0}=\bar{a} b_{0}-d \bar{b}_{n+1}+\bar{b} b_{1}-c \bar{b}_{n}, \\
\beta a_{k-1}=\bar{a} b_{k-1}-d \bar{b}_{n-k+2}+\bar{b} b_{k}-c \bar{b}_{n-k+1}, \quad k=2, \ldots, n, \\
\beta a_{n}=\bar{a} b_{n}-d \bar{b}_{1}+\bar{b} b_{n+1}-c \bar{b}_{0}, \\
0=\bar{a} b_{n+1}-d \bar{b}_{0} .
\end{gathered}
$$


Observe that the first and the last equations in (2.19) coincide with (2.18). According to (2.17) and (2.18), one obtains the following relation for the coefficient $\beta$ of the polynomial $g(z)$ :

$$
\beta=\left(\left|b_{n+1}\right|^{2}-\left|b_{0}\right|^{2}\right)\left(\frac{|c|^{2}}{\left|b_{0}\right|^{2}}-\frac{|d|^{2}}{\left|b_{n+1}\right|^{2}}\right) \neq 0
$$

Thus the coefficients $a_{0}, a_{1}, \ldots, a_{n}$ of the polynomial $f$ can be uniquely determined from system (2.19).

It follows from Theorems 2.1 and 2.2 that the existence of a regular point for the polynomial $F$ is a sufficient condition for the existence of a solution to the functional equation (1.3).

It turns out that the existence of a regular point for the polynomial $F$ is intimately connected to the linear (in)dependence of the polynomials $F$ and $F^{*}$ in the complex linear space of (complex) polynomials. To be more precise, there exists a regular point for $F$ if and only if $F$ and $F^{*}$ are linearly independent. This statement is a direct consequence of the following lemma.

LEMMA 2.3. The following conditions are equivalent:

(a) $F$ and $F^{*}$ are linearly independent in the complex linear space of (complex) polynomials;

(b) the identity $|F(z)| \equiv\left|F^{*}(z)\right|$ is satisfied;

(c) the identity

$$
2 \operatorname{Re}\left[\overline{F(w)} F^{\prime}(w) w\right] \equiv(n+1)|F(w)|^{2} \quad \forall|w|=1
$$

is satisfied.

Proof. Assume (a) is satisfied: $F^{*}=C \cdot F$ for some nonzero complex number $C$. Then, according to the definition of the polynomial $F^{*}$, one has the following equality for the coefficients $b_{0}, b_{n+1}: \bar{b}_{0}=C b_{n+1}, \bar{b}_{n+1}=C b_{0}$, from which it follows that $|C|=1$, and therefore, $\left|F^{*}(z)\right| \equiv|F(z)|$.

Thus (a) implies (b).

Assume, further, $|F(z)| \equiv\left|F^{*}(z)\right|$. Applying to this identity the change of coordinates $z=r w, r \geq 0,|w|=1$ and using the definition of $F^{*}$, one obtains

$$
r^{2 n+2} F\left(r^{-1} w\right) \overline{F\left(r^{-1} w\right)} \equiv F(r w) \overline{F(r w)}
$$

Differentiating the last identity with respect to the real argument $r$ at the point $r=1$ we obtain (c).

Thus (b) implies (c).

Finally, assume that (c) is satisfied and show that $F$ and $F^{*}$ are linearly dependent. Consider the function $\overline{F(w)} / F(w)$ on the unit circle $|w|=1$, where $F(w) \neq 0$, and show that this function has a continuous extension over the unit circle. Assume that $F$ vanishes at some point $w_{0}$ belonging to the unit circle. Then we have the following representation: $F(z)=\left(z-w_{0}\right)^{k} F_{1}(z)$, where $k \geq 1$ is an integer, $F_{1}\left(w_{0}\right) \neq 0$. 
8 On a certain functional equation in the algebra of polynomials

The conditions

$$
\lim _{w \rightarrow w_{0}} \frac{\bar{w}-\bar{w}_{0}}{w-w_{0}}=-\frac{1}{w_{0}^{2}}, \quad|w|=1,
$$

yield

$$
\lim _{w \rightarrow w_{0}} \frac{\overline{F(w)}}{F(w)}=(-1)^{k} w_{0}^{-2 k} \frac{\overline{F_{1}\left(w_{0}\right)}}{\overline{F_{1}\left(w_{0}\right)}}, \quad|w|=1,
$$

providing the continuity of the function $\overline{F(w)} / F(w)$.

Differentiating the function $w^{n+1} \overline{F(w)} / F(w), w=\exp (i t)$ with respect to $t$ at the points where $F(w) \neq 0$ and using condition (c), one obtains

$$
\frac{d}{d t} \frac{w^{n+1} \overline{F(w)}}{F(w)}=i w^{n+1}\left\{\frac{(n+1) \overline{F(w)}-\overline{F^{\prime}(w) w}}{F(w)}-\frac{\overline{F(w)} F^{\prime}(w) w}{F^{2}(w)}\right\}=0 .
$$

The above equality along with the continuity of the function $w^{n+1} \overline{F(w)} / F(w)$ on the unit circle yield that the latter function is, in fact, constant, that is $w^{n+1} \overline{F(w)}=C \cdot F(w)$ or, equivalently, $F^{*}(w)=C \cdot F(w),|w|=1$. Therefore, the coefficients of the polynomials $C \cdot F$ and $F^{*}$ coincide. The linear dependence of the polynomials $F$ and $F^{*}$ is established and the proof of Lemma 2.3 is complete.

\section{An algorithm for computing the number of roots in the unit circle}

In what follows we will be interested in the case when the coefficients $a, b, c, d$ satisfy the following additional condition:

$$
|a+b w| \geq|c+d w|, \quad|w|=1
$$

LeMma 3.1. Linear functions $a+b z, c+d z$ satisfy condition (3.1) if and only if the numbers $\alpha=\bar{a} b-\bar{c} d, \beta=|a|^{2}+|b|^{2}-|c|^{2}-|d|^{2}$ satisfy the inequality

$$
2|\alpha| \leq \beta
$$

Proof. The equality

$$
|a+b z|^{2}-|c+d z|^{2}=|a|^{2}+2 \mathfrak{R}(\bar{a} b z)+|b|^{2}|z|^{2}-|c|^{2}-2 \mathfrak{R}(\bar{c} d z)-|d|^{2}|z|^{2}
$$

yields, for $z=e^{i t}, t \in[0,2 \pi]$,

$$
|a+b z|^{2}-|c+d z|^{2}=\beta+2 \Re\left[\alpha e^{i t}\right] .
$$

Combining this with the equality

$$
\min _{t} \mathfrak{R}\left[\alpha e^{i t}\right]=-|\alpha|
$$

one obtains the equivalence of conditions (3.1) and (3.2). The lemma is proved. 
Observe that Lemma 3.1 allows one to verify effectively the validity of condition (3.1) for the coefficients $a, b, c, d$ determined by regular points of the polynomial $F$.

Lemma 3.2. Let $z_{0},\left|z_{0}\right| \leq 1$, be a regular point of the polynomial F. Assume that the numbers $a, b, c, d$ are determined by equalities (2.5) with

$$
\left(\left|A_{0}\right|-1\right)\left(\left|\sigma_{0} A_{0}\right|-\left|\sigma_{1}\right|\right)>0, \quad\left|z_{0}\right|<1,
$$

or that they satisfy (2.12) with

$$
\Delta_{0}\left[\Delta_{0}\left|c+z_{0} d\right|^{2}-\left|F\left(z_{0}\right)\right|^{2}\left(|c|^{2}-|d|^{2}\right)\right]>0, \quad\left|z_{0}\right|=1
$$

Then $a, b, c, d$ satisfy (3.1).

The statement following below provides an important property of solution $(a, b, c, d, f)$ to the functional equation (1.3).

Theorem 3.3. Assume that the polynomial $F$ does not contain roots on the unit circle $|z|=$ 1. Suppose, further, that the coefficients $a, b, c, d$ satisfy condition (3.1) and

$$
|a d-b c|+\beta>0, \quad \beta=|a|^{2}+|b|^{2}-|c|^{2}-|d|^{2} .
$$

Then the polynomial $f$ as well as any polynomial of the parameterized family

$$
G_{\lambda}(z)=(a+b z) f(z)+\lambda(c+d z) f^{*}(z), \quad 0 \leq \lambda \leq 1,
$$

does not contain roots on the unit circle $|z|=1$.

Proof. Arguing indirectly, one obtains the existence of numbers $w,|w|=1$, and $\lambda \in[0,1]$ such that

$$
G_{\lambda}(w)=(a+b w) f(w)+\lambda(c+d w) f^{*}(w)=0 .
$$

Since $\bar{w} w=1$, one has

$$
f^{*}(w)=w^{n} \overline{f\left(\frac{1}{\bar{w}}\right)}=w^{n} \overline{f(w)}
$$

from which it follows that

$$
(a+b w) f(w)+\lambda(c+d w) w^{n} \overline{f(w)}=0 .
$$

By condition,

$$
G_{1}(w)=(a+b w) f(w)+(c+d w) w^{n} \overline{f(w)}=F(w) \neq 0
$$

therefore, $f(w) \neq 0$ and $0 \leq \lambda<1$. Now, using the equality (3.12), we obtain

$$
|a+b w|=\lambda|c+d w|
$$


from which it follows (see Lemma 3.1) that $|a+b w|=0,|c+d w|=0$. The latter equalities yield $|a|=|b|,|c|=|d|, a d-b c=0$ that contradicts condition (3.8) and the result follows.

Denote by $\kappa(F)$ the number of roots of the polynomial $F$ (counted according to their multiplicity) belonging to the open unit disc $|z|<1$. As noted, the computing $\kappa(F)$ maybe a reduction to the generalized Rauss-Hurwitz problem of determining the number of roots of a polynomial in a semiplane (see, for instance, [1-3]). The above results allow us to construct an iterative process for computing $\kappa(F)$, namely, the following theorem.

Theorem 3.4. Assume that the polynomial $F$ does not have roots on the unit circle $|z|=1$. If the polynomials $F$ and $F^{*}$ are linearly dependent, then

$$
\kappa(F)=\frac{n+1}{2}
$$

otherwise

$$
\kappa(F)=\kappa\left(G_{0}\right)
$$

where $G_{0}(z)=(a+b z) f(z)$ and the collection $(a, b, c, d, f)$ satisfying (3.1), (3.8) is a solution to the functional equation (1.3).

Proof. Assume the polynomials $F$ and $F^{*}$ to be linearly dependent, that is $F^{*}(z) \equiv C F(z)$. Then the following presentation takes place:

$$
F(z)=b_{n+1}\left(z-z_{1}\right)^{\alpha_{1}} \cdots\left(z-z_{m}\right)^{\alpha_{m}}\left(z-\frac{1}{\bar{z}_{1}}\right)^{\alpha_{1}} \cdots\left(z-\frac{1}{\bar{z}_{m}}\right)^{\alpha_{m}}
$$

where

$$
\left|z_{s}\right|<1, \quad s=1, \ldots, m, \quad 2\left(\alpha_{1}+\cdots+\alpha_{m}\right)=n+1 .
$$

From this it follows that the number $n+1$ is even and

$$
\kappa(F)=\alpha_{1}+\cdots+\alpha_{m}=\frac{(n+1)}{2} .
$$

Assume now the polynomials $F$ and $F^{*}$ to be linearly independent. Then, by Lemma 2.3, $F$ admits a regular point $z_{0},\left|z_{0}\right| \leq 1$. Therefore, by Lemmas 1.1, 2.3, and 3.2, there exists a collection $(a, b, c, d, f)$ satisfying conditions (3.1), (3.8) and being a solution to the functional equation (1.3). Combining Theorem 3.3 and the Rouche theorem one obtains $\kappa(F)=\kappa\left(G_{0}\right)$, where $G_{0}(z)=(a+b z) f(z)$.

The proof of Theorem 3.4 is complete.

It is easy to see that the number $\kappa\left(G_{0}\right)$ satisfies the equality

$$
\kappa\left(G_{0}\right)=\kappa(f)+\varepsilon,
$$

where $\varepsilon=1$ for $|a|<|b|$ and $\varepsilon=0$, otherwise. A simple argument shows that if the numbers $a, b, c, d$ are determined by a regular point $z_{0}$, then $\varepsilon$ can be evaluated according to 
the formulae

$$
\varepsilon=\frac{1+\operatorname{sign}\left(\left|F^{*}\left(z_{0}\right)\right|-\left|F\left(z_{0}\right)\right|\right)}{2}, \quad\left|z_{0}\right|<1, \quad \varepsilon=\frac{1-\operatorname{sign}\left(\Delta_{0}\right)}{2}, \quad\left|z_{0}\right|=1 .
$$

Thus, under the assumptions of Theorems 2.1 and 2.2, one has

$$
\begin{gathered}
\kappa(F)=\frac{1+\operatorname{sign}\left(\left|F^{*}\left(z_{0}\right)\right|-\left|F\left(z_{0}\right)\right|\right)}{2}+\kappa(f), \quad\left|z_{0}\right|<1, \\
\kappa(F)=\frac{1-\operatorname{sign}\left(\Delta_{0}\right)}{2}+\kappa(f), \quad\left|z_{0}\right|=1 .
\end{gathered}
$$

Formulae (3.22) and (3.23) give rise to a recurrent procedure for the computation of $\kappa$. Indeed, they allow one to compute $\kappa(F)$, where $F$ is a polynomial of degree $n+1$, based on $\kappa(f)$, where $f$ is a polynomial of degree $n$ and its coefficients are completely determined by coefficients of $F$.

Observe that if $z_{0}=0$, then formula (3.22) takes the form

$$
\kappa(F)=\frac{1+\operatorname{sign}\left(\left|b_{n+1}\right|-\left|b_{0}\right|\right)}{2}+\kappa(f) .
$$

Here the coefficients of $f$ can be determined from system (2.19).

\section{Criterion for the absence of roots on the unit circle}

Given the coefficients of the polynomials $F$ and $F^{*}$, one can construct the following $(2 n+$ $2) \times(2 n+2)$ matrix:

$$
M_{F}=\left(\begin{array}{cccccc}
b_{0} & b_{1} & \cdot & b_{n+1} & \cdot & 0 \\
\bar{b}_{n+1} & \bar{b}_{n} & \cdot & \bar{b}_{0} & \cdot & 0 \\
0 & b_{0} & \cdot & b_{n} & \cdot & 0 \\
0 & \bar{b}_{n+1} & \cdot & \bar{b}_{0} & \cdot & 0 \\
\cdot & \cdot & \cdot & \cdot & \cdot & \cdot \\
0 & 0 & \cdot & b_{1} & \cdot & b_{n+1} \\
0 & 0 & \cdot & \bar{b}_{n+1} & \cdot & \bar{b}_{0}
\end{array}\right) .
$$

The matrix $M_{F}$ coincides with the Sylvester matrix of $F$ and $F^{*}$ (up to a permutation of its lines). Therefore, $\operatorname{det}\left(M_{F}\right)$ coincides (up to a sign) with the resultant $R\left(F, F^{*}\right)$ of the polynomials $F$ and $F^{*}$. Let $z_{s}, s=1, \ldots, n+1$ be all the roots of $F$ (counted according to their multiplicities). By condition, $F(0)=b_{0} \neq 0$, therefore, all the roots are different from zero. By definition of the polynomial $F^{*}$, the numbers $\bar{z}_{s}^{-1}, s=1, \ldots, n+1$, are roots of $F^{*}$. Hence (cf. [5]), $R\left(F, F^{*}\right)$ can be represented as follows:

$$
R\left(F, F^{*}\right)=\bar{b}_{0}^{n+1} b_{n+1}^{n+1} \prod_{s, t}\left(z_{s}-\frac{1}{\bar{z}_{t}}\right) .
$$

Formula (4.2) gives rise to the following criteria for the polynomial $F$ to have no roots on the unit circle. 
Theorem 4.1. Let $\operatorname{det}\left(M_{F}\right)$ be different from zero. Then $F$ does not have roots on the unit circle $|z|=1$.

Assume that a collection $(a, b, 1,1, f)$ of the numbers $a, b, c=d=1$ and a polynomial $f$ satisfy the functional equation (1.3) and the condition $\alpha \equiv \bar{a} b-1=0$. Using $a, b$ define the following square matrix of order $2 n+2$ :

$$
P=\left(\begin{array}{ccccccccc}
1 & 0 & 0 & 0 & \cdot & 0 & 0 & 0 & 0 \\
-\bar{b} & 1 & -\bar{a} & 1 & \cdot & 0 & 0 & 0 & 0 \\
1 & -a & 1 & -b & \cdot & 0 & 0 & 0 & 0 \\
0 & 0 & -\bar{b} & 1 & \cdot & 0 & 0 & 0 & 0 \\
0 & 0 & 1 & -a & \cdot & 0 & 0 & 0 & 0 \\
\cdot & \cdot & \cdot & \cdot & \cdot & \cdot & \cdot & \cdot & \cdot \\
0 & 0 & 0 & 0 & \cdot & -\bar{b} & 1 & -\bar{a} & 1 \\
0 & 0 & 0 & 0 & \cdot & 1 & -a & 1 & -b \\
0 & 0 & 0 & 0 & \cdot & 0 & 0 & 0 & 1
\end{array}\right) .
$$

It is easy to see that $\operatorname{det}(P)$ satisfies the following condition:

$$
\operatorname{det} P=(-1)^{n-1} \beta^{n-1}\left(1-|a|^{2}\right),
$$

where $\beta=|a|^{2}+|b|^{2}-2$.

Let $M_{f}$ be a square matrix of order $2 n$ determined by the polynomial $f$. Using the matrices $M_{F}, M_{f}$, and $P$ one can express a connection between the coefficients of polynomials $F$ and $f$, given in system (2.19), by the following matrix equality:

$$
P M_{F}=\left(\begin{array}{ccccccc}
b_{0} & b_{1} & b_{2} & \cdot & & & 0 \\
0 & & & & & \\
0 & & & \beta M_{f} & & & 0 \\
\cdot & & & & & & \cdot \\
0 & & & \cdot & \bar{b}_{2} & \bar{b}_{1} & \bar{b}_{0}
\end{array}\right) .
$$

Consider the matrix written in the right-hand side of (4.5) more intently: (i) its first and last lines coincide with the corresponding lines of the matrix $M_{F}$; (ii) the remaining entries (except for zeros related to the first and the last column) are filled out by the elements of the matrix $\beta \cdot M_{F}$. Therefore, equality (4.5) connects $\operatorname{det}\left(M_{F}\right)$ and $\operatorname{det}\left(M_{f}\right)$ as follows.

Theorem 4.2. Let $c=d=1$ and assume that the numbers $a, b$ along with coefficients $a_{0}, \ldots, a_{n}$ of the polynomial $f$ satisfy (2.19), $\beta \neq 0$. Then one has

$$
\operatorname{det} M_{F}=(-1)^{n+1}\left(\left|b_{n+1}\right|^{2}-\left|b_{0}\right|^{2}\right) \beta^{n} \operatorname{det} M_{f}
$$

Proof. Combining equality (4.5), the above formula for $\operatorname{det}(P)$ with the standard determinant properties yields

$$
\operatorname{det} M_{F}=\frac{\left|b_{0}\right|^{2} \operatorname{det}\left(\beta M_{f}\right)}{\operatorname{det} P}=(-1)^{n+1}\left(\left|b_{n+1}\right|^{2}-\left|b_{0}\right|^{2}\right) \beta^{n} \operatorname{det} M_{f} .
$$


Assume a collection $(a, b, 1,1, f, F)$ of the numbers $a, b, c=d=1$ and polynomials $f, F$ to satisfy the functional equation (1.3) and the condition $\alpha \equiv \bar{a} b-1=0$. Consider a sequence of collections $\left(a^{k}, b^{k}, 1,1, F^{k}\right)$ of numbers $a^{k}, b^{k}, c^{k}=d^{k}=1$ and polynomials

$$
F^{k}(z)=b_{0 k}+b_{1 k} z+\cdots+b_{k k} z^{k}, \quad b_{0 k} \neq 0, b_{k k} \neq 0
$$

of degree $k$, satisfying the functional equation

$$
\left(a^{k}+b^{k} z\right) F^{k}(z)+(1+z)\left(F^{k}\right)^{*}(z)=F^{k+1}(z), \quad k=n, n-1, \ldots, 1,
$$

and the condition

$$
\alpha^{k} \equiv \overline{a^{k}} b^{k}-1=0,
$$

where $a^{n}=a, b^{n}=b F^{n}=f, F^{n+1}=F$. By Theorem 2.1, if $z=0$ is a regular point of the polynomial

$$
F^{k+1}(z)=b_{0 k+1}+b_{1 k+1} z+\cdots+b_{k+1, k+1} z^{k+1}
$$

that is,

$$
b_{0 k+1} \cdot b_{k+1, k+1} \neq 0, \quad\left|b_{0 k+1}\right| \neq\left|b_{k+1, k+1}\right|
$$

then the system of (4.9), (4.10) has the unique solution $\left(a^{k}, b^{k}, F^{k}\right)$. Moreover,

$$
\overline{a^{k}}=\frac{\overline{b_{0 k+1}}}{b_{k+1, k+1}}, \quad \overline{b^{k}}=\frac{1}{a^{k}} .
$$

This along with formula (3.24) justify the following relation:

$$
\kappa(F)=\sum_{k=1}^{n+1} \frac{1+\operatorname{sign}\left(\left|b_{k k}\right|-\left|b_{0 k}\right|\right)}{2} .
$$

\section{Application for computing the rotation of a plane vector field}

(1) Consider a vector field $\Phi(x, y)=\{p(x, y), q(x, y)\}$, where

$$
p(x, y)=\sum_{k, j} a_{k j} x^{k} y^{j}, \quad q(x, y)=\sum_{k, j} b_{k j} x^{k} y^{j}
$$

are polynomials in real variables $x$ and $y$ with real coefficients. Assume that $\Phi(x, y) \neq 0$, $(x, y) \in S=\left\{(x, y): x^{2}+y^{2}=1\right\}$. We are interested in computing the rotation $\gamma(\Phi, S)$.

Recall the definition of rotation. Consider the complex presentation of the field $\Phi$ :

$$
p+i q=\exp (i \theta(t))|p+i q|, \quad p+i q=p(\cot t, \sin t)+i q(\cos t, \sin t), \quad t \in[0,2 \pi],
$$


14 On a certain functional equation in the algebra of polynomials

where $\theta(t)$ is a continuous function. Then (cf. [4])

$$
\gamma(\Phi, S):=\frac{1}{2 \pi}(\theta(2 \pi)-\theta(0))
$$

The following statement reduces the computation of rotation of a plane vector field to the computation of the number of roots in the unit disc of some polynomial.

Lemma 5.1. Given a (polynomial) plane vector field $\Phi$, there exists a unique pair $(m, F)$, where $m$ is an integer and $F$ is a polynomial in complex variable with complex coefficients, $F(0) \neq 0$, satisfying the following condition:

$$
F(z)=z^{m}(p(x, y)+i q(x, y)), \quad z=x+i y,|z|=1 .
$$

Proof. Take a polynomial $P(x, y)=p(x, y)+i q(x, y)$ in real variables $x, y$. The change of variables

$$
(x, y) \longrightarrow\left(\frac{1+z^{2}}{2 z}, \frac{i\left(1-z^{2}\right)}{2 z}\right)
$$

determines the rational function in complex variable $z$ :

$$
R(z)=P\left(\frac{1+z^{2}}{2 z}, \frac{i\left(1-z^{2}\right)}{2 z}\right)
$$

The function $R(z)$ can be represented in the form

$$
R(z)=\frac{F(z)}{z^{m}}
$$

where $F(z)$ is a polynomial satisfying the condition $F(0) \neq 0$ and $m$ is an integer. Since

$$
x=\frac{1+z^{2}}{2 z}, \quad y=\frac{i\left(1-z^{2}\right)}{2 z}, \quad z=x+i y,|z|=1,
$$

the pair $(m, F)$ satisfies $(5.4)$.

To complete the proof of Lemma 5.1, it remains to establish the uniqueness of the pair satisfying (5.4). Suppose that $\left(m_{1}, F_{1}\right)$ is another pair satisfying

$$
F_{1}(z)=z^{m_{1}}(p(x, y)+i q(x, y)), \quad z=x+i y,|z|=1,
$$

and $F_{1}(0) \neq 0$. Assuming, without loss of generality, that $m_{1} \geq m$, one obtains the following equalities for $F_{1}$ and $z^{m_{1}-m} F$ :

$$
F_{1}(z)=z^{m_{1}} P(x, y)=z^{m_{1}-m} z^{m} P(x, y)=z^{m_{1}-m} F(z)
$$

for $z=x+i y,|z|=1$. From this it follows that $F_{1}$ and $z^{m_{1}-m} F$ coincide. Further, by assumption, $F(0) \neq 0, F_{1}(0) \neq 0$, hence $m_{1}=m$. Thus $F_{1}=F$ and Lemma 5.1 is completely proved. 
Let $\Phi$ and $F$ be as in Lemma 5.1. Then $\gamma(\Phi, S)$ and $\kappa(F)$ satisfy the following relation:

$$
\kappa(F)=m+\gamma(\Phi, S)
$$

(2) Assume a field $\Phi$ to be given in a parametric form: $\Phi(t)=\{p(t), q(t)\}$, where $p(t)$, $q(t)$ are real continuous $2 \pi$-periodic functions. Suppose $\Phi(t) \neq 0, t \in[0,2 \pi]$. The field $\Phi$ may be considered as the one defined on the unit circle $S$, by assigning to each point $x=$ $\cos t, y=\sin t$ the vector $\{p(t), q(t)\}$. Therefore, the rotation $\gamma(\Phi, S)$ is correctly defined on $S$. Assuming the functions $p(t), q(t)$ to be smooth enough, one can assign to the field $\Phi$ the Fourier series of the complex function $P(t)=p(t)+i q(t)$ :

$$
P(t)=\sum_{k=-\infty}^{\infty} c_{k} \exp (i k t) .
$$

Since the series (5.12) converges uniformly and $\Phi$ does not vanish, there exists an integer $N$ such that for all $t \in[0,2 \pi]$ the following estimate is true:

$$
\left|P(t)-\sum_{k=-N}^{N} c_{k} \exp (i k t)\right|<|P(t)| .
$$

Set $m:=\max \left\{-k:\left|c_{k}\right|>0,|k| \leq N\right\}$ and consider the polynomial

$$
F(z)=\sum_{k=0}^{N+m} c_{k-m} z^{k} .
$$

Using the same arguments as in Section 1 it is easy to check that $\gamma(\Phi, S)$ and $\kappa(F)$, where $F$ is defined by (5.14), satisfy (5.11).

\section{References}

[1] N. G. Chebotarev and N. N. Meyman, Rauss-Hurwitz stability problem for polynomials and entire functions, Trudy Matematicheskogo Instituta Imeni V. A. Steklova 26 (1949).

[2] B. P. Demidovič, Lectures on the Mathematical Theory of Stability, Izdat. "Nauka", Moscow, 1967.

[3] F. R. Gantmaher, Matrix Theory, Nauka, Moscow, 1967.

[4] M. A. Krasnosel'sky, A. I. Perov, A. I. Povolotsky, and P. P. Zabreiko, Plane Vector Fields, Fizmatgiz, Moscow, 1961.

[5] V. V. Prasolov, Polynomials, MCNMO, Moscow, 2003.

E. Muhamadiev: Department of Information Systems Technologies, Vologda State Technical University, 15 Lenin Street, Vologda 160035, Russia

E-mail address: muhamerg@mail.vstu.edu.ru 


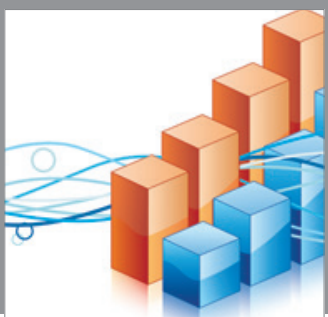

Advances in

Operations Research

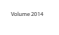

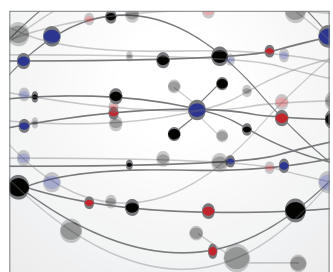

\section{The Scientific} World Journal
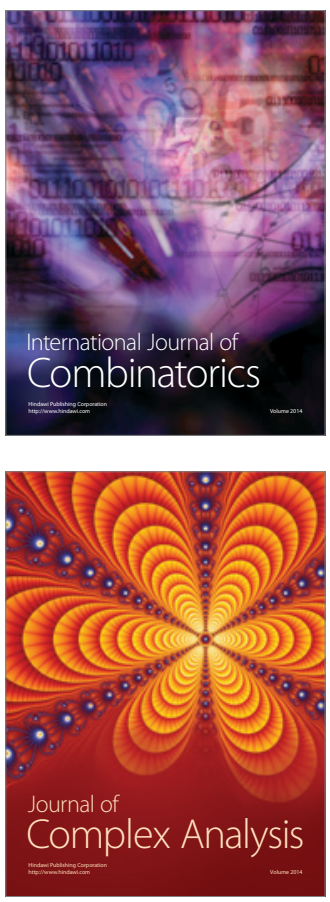

International Journal of

Mathematics and

Mathematical

Sciences
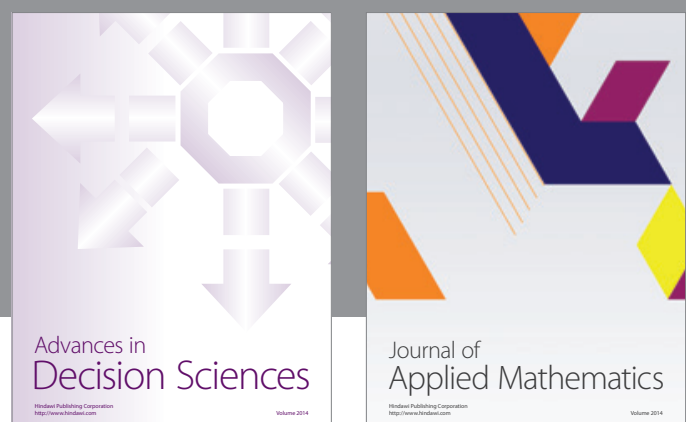

Journal of

Applied Mathematics
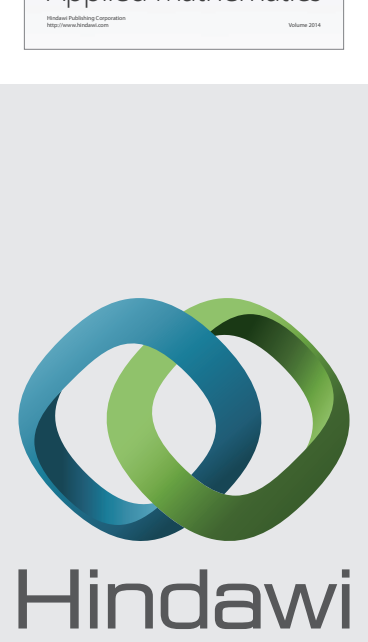

Submit your manuscripts at http://www.hindawi.com
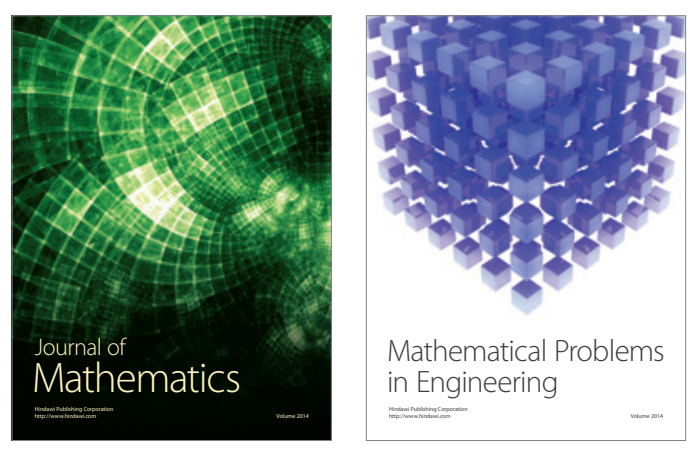

Mathematical Problems in Engineering
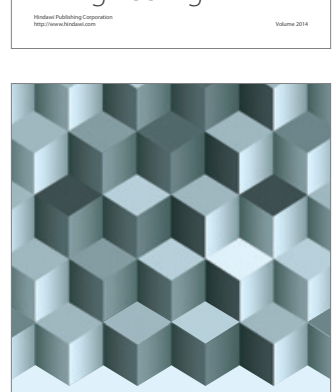

Journal of

Function Spaces
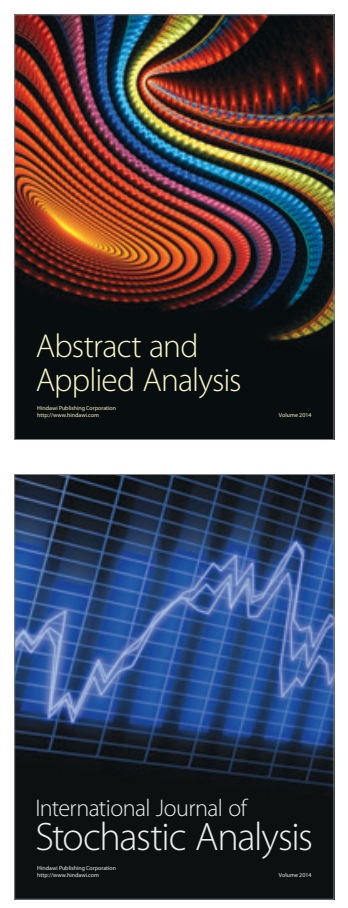

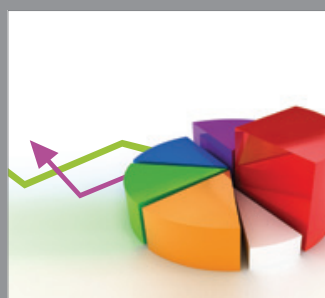

ournal of

Probability and Statistics

Promensencen
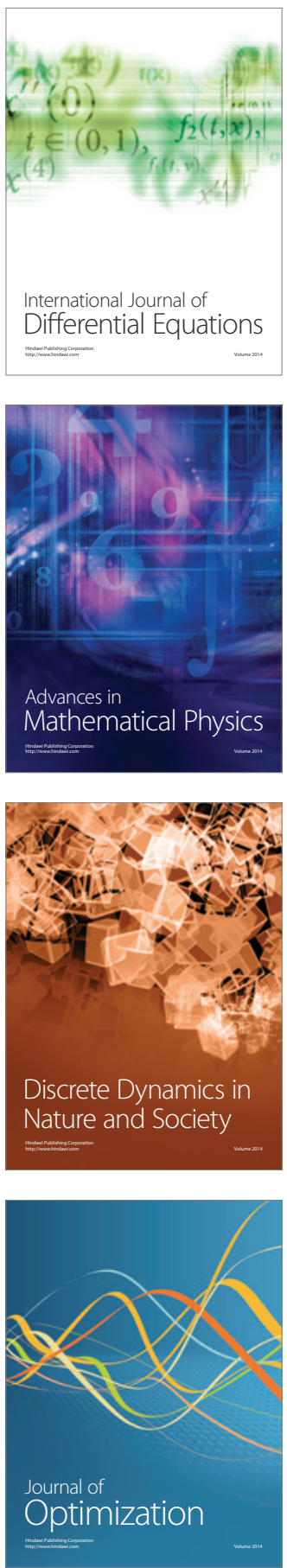